\title{
Uplink Capacity of MIMO Cellular Systems with Multicell Processing
}

\author{
Symeon Chatzinotas, Muhammad Ali Imran, Costas Tzaras \\ Centre for Communication Systems Research \\ University of Surrey, United Kingdom, GU2 7XH \\ \{S.Chatzinotas, M.Imran, C.Tzaras\}@surrey.ac.uk
}

\begin{abstract}
Multiple antennas are known to increase the link throughput by providing a multiplexing gain which scales with the number of antennas. Especially in cellular systems, multiple antennas can be exploited to achieve higher rates without the need for additional Base Station (BS) sites. In this direction, this paper investigates the multi-antenna capacity scaling in a cellular system which employs multicell processing (hyperreceiver). The model under investigation is a MIMO Gaussian Cellular Multiple-Access Channel (GCMAC) over a planar cellular array in the presence of power-law path loss and flat fading. Furthermore, the considered cellular model overcomes the assumption of user collocation utilized by previous models by incorporating uniformly distributed User Terminals (UTs). The asymptotic eigenvalue distribution (a.e.d.) of the covariance channel matrix is calculated based on free-probabilistic arguments. In this context, we evaluate the effect of multiple BS/UT antennas on the optimal sum-rate capacity by considering a variable-density cellular system. Finally, the analytical results are interpreted in the context of a typical real-world macrocellular scenario.
\end{abstract}

\section{INTRODUCTION}

Across the information-theoretic literature, it has been widely proven that multiple antennas are able to increase the link throughput by providing a multiplexing gain which scales with the number of antennas. In the context of multiuser channels, the sum-rate capacity of a MIMO multiple-access channel was investigated in [1], [2] and [3]. In this direction, it was shown that the sum capacity with perfect Channel State Information available at the receiver (CSIR) scales linearly with $\min \left(n_{r}^{t o t}, n_{t}^{t o t}\right)$, where $n_{r}^{\text {tot }}$ and $n_{t}^{\text {tot }}$ is the total number of receive and transmit antennas respectively.

In the research area of the Gaussian Cellular Multiple Access Channel (GCMAC), the first concrete result for the single antenna case was presented by Wyner in [4]. Using a very simple but tractable model for the cellular uplink channel, Wyner showed the importance of joint decoding at the Base Station (BS) receivers (hyper-receiver) and found the analytical formulas of the maximum sum-rate capacity under the assumption of multicell processing. This model triggered the interest of the research community in the cellular capacity limits and it was subsequently extended for flat fading environments [5]. In addition, [3] has investigated the capacity scaling in the asymptotic regime where the number of BS antennas and the number of UTs grow large. Subsequently, authors in [6] have analyzed the capacity performance for both uplink and downlink linear Wyner-like models with UTs collocated at the cell-edge. The reader is referred to [7] and references therein for a more complete literature review. One major assumption shared amongst the afomentioned models is that the cell density is fixed and only physically adjacent cells interfere. The author in [8], extended the Wyner's model by assuming multiple-tier interference and incorporated a distancedependent path loss factor in order to study the effect of cell density in a variable cell-density linear cellular array. However, the assumption of user collocation was still maintained to keep the model tractable.

In this paper, we further extend these models in order to incorporate the effect of user distribution in combination with multiple antennas. Instead of assuming collocated UTs, we assume that UTs are spatially distributed within the cell and each channel gain is affected by a distance-dependent path loss factor. The effect of multiple BS and UT antennas is evaluated and discussed in the context of a MIMO Gaussian Cellular Multiple-Access Channel (GCMAC) over a planar cellular array in the presence of power-law path loss and flat fading. Throughout the formulations of this paper, $\mathbb{E}[\cdot]$ denotes the expectation, $(\cdot)^{*}$ denotes the complex conjugate, $(\cdot)^{\dagger}$ denotes the conjugate transpose matrix, $\odot$ denotes the Hadamard product and $\otimes$ denotes the Kronecker product. The logarithmic expression $\log (x)$, unless explicitly stated otherwise, refers to the natural $\operatorname{logarithm} \log _{e}(x)$. The figure of merit studied herewith is the per-cell sum-rate capacity achieved with multicell joint decoding and it is denoted by $C_{\text {opt }}$.

The rest of the paper is organised as follows. In the next section, we describe the proposed model and the derivation of the information theoretic capacity of the cellular system. In section III, we evaluate and compare the capacity results produced by both simulation and analysis. Furthermore, section IV interprets the analytical results in the context of a typical macrocellular scenario. The last section discusses the presented results from a system-design point of view and concludes the paper.

\section{Model Description AND AnAlysis}

Assume that $K$ UTs are uniformly distributed in each cell of a planar cellular system comprising $N$ base stations and that each BS and each UT are equipped with $n_{B S}$ and $n_{U T}$ antennas respectively. The received signal at cell $n$, at time 
index $t$, is given by:

$$
\mathbf{y}^{n}[t]=\sum_{m=1}^{N} \sum_{k=1}^{K} \varsigma_{k}^{n m} \mathbf{G}_{k}^{n m}[t] \mathbf{x}_{k}^{m}[t]+\mathbf{z}^{n}[t]
$$

where $\mathbf{x}_{k}^{m}[t]$ is the $t$ th complex channel symbol vector $n_{U T} \times 1$ transmitted by the $k$ th UT of the $m$ th cell and $\left\{\mathbf{G}_{k}^{n m}\right\}$ is a $n_{U T} \times n_{U T}$ random matrix with independent, strictly stationary and ergodic complex c.c.s. i.i.d. elements. The matrix $\mathbf{G}_{k}^{n m}[t]$ represents the multiple-antenna correlated flat fading processes experienced in the transmission path between the $n_{B S}$ receive antennas of the $n$th $\mathrm{BS}$ and the $n_{U T}$ transmit antennas of the $k$ th UT in the $m$ th cell. The fading coefficients are assumed to have unit power, i.e. $\mathbb{E}\left[\mathbf{G}_{k}^{n m}[t] \mathbf{G}_{k}^{n m}[t]^{\dagger}\right]=\mathbf{I}$ for all $(n, m, k)$ and all UTs are subject to a power constraint $P$, i.e. $\mathbb{E}\left[\operatorname{tr}\left(\boldsymbol{x}_{k}^{m}[t] \boldsymbol{x}_{k}^{m}[t]^{\dagger}\right)\right] \preceq P$ for all $(m, k)$. It should be noted that the UTs are assumed to be totally ignorant of the channel state information (CSI). In case the UTs had perfect of even statistical CSI, input optimization strategies could be used to maximize the ergodic capacity [9], [10] . However, in our case the optimal transmission strategy is to uniformly allocate the UT power $P$ across the $n_{U T}$ transmit antennas i.e. $\mathbb{E}\left[\boldsymbol{x}_{k}^{m}[t] \boldsymbol{x}_{k}^{m}[t]^{\dagger}\right]=\frac{P}{n_{U T}} \mathbf{I}_{n_{U T}}$. The variance coefficients $\varsigma_{k}^{n m}$ in the transmission path between the $m$ th BS and the $k$ th UT in the $n$th cell are calculated according to the "modified" power-law path loss model [8], [11]:

$$
\varsigma_{k}^{n m}=\left(1+d_{k}^{n m}\right)^{-\eta / 2},
$$

where $\eta$ denotes the path loss exponent. Dropping the time index $t$, the aforementioned model can be compactly expressed as a vector memoryless channel of the form $\mathbf{Y}=\mathbf{H X}+\mathbf{Z}$, where the vector $\mathbf{Y}=\left[\mathbf{y}^{(1)} \ldots \mathbf{y}^{(N)}\right]^{T}$ with $\mathbf{y}^{(n)}=\left[y^{1} \ldots y^{n_{B S}}\right]$ represents received signals by the $\mathrm{BSs}$, the vector $\mathbf{X}=$ $\left[\mathbf{x}_{(1)}^{(1)} \ldots \mathbf{x}_{(K)}^{(N)}\right]^{T}$ with $\mathbf{x}_{(k)}^{(n)}=\left[x^{1} \ldots y^{n_{U T}}\right]$ represents transmit signals by all the UTs of the cellular system and the compo-

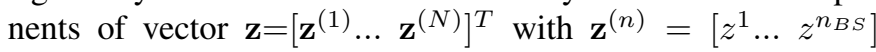
are i.i.d c.c.s. random variables representing AWGN with $\mathbb{E}\left[\mathbf{z}^{n}\right]=\mathbf{0}, \quad \mathbb{E}\left[\mathbf{z}^{n}[t] \mathbf{z}^{n}[t]^{\dagger}\right]=\sigma^{2} \mathbf{I}$. The channel matrix can be rewritten as:

$$
\mathbf{H}=\boldsymbol{\Sigma}_{\mathbf{M}} \odot \mathbf{G}_{\mathbf{M}}
$$

where $\mathbf{G}_{M} \sim \mathcal{C N}\left(\mathbf{0}, \mathbf{I}_{N n_{B S}}\right)$ is a complex Gaussian $N n_{B S} \times$ $K N n_{U T}$ matrix, comprising the Rayleigh fading coefficients between the $K N n_{U T}$ transmit and the $N n_{B S}$ receive antennas. Similarly, $\boldsymbol{\Sigma}_{\mathbf{M}}$ is a $N n_{B S} \times K N n_{U T}$ deterministic matrix, comprising the path loss coefficients between the $K N n_{U T}$ transmit and the $N n_{B S}$ receive antennas. Since the multiple antennas of each UT / BS are collocated, $\boldsymbol{\Sigma}_{\mathbf{M}}$ can be written in the form of a block matrix based on the variance profile matrix $\boldsymbol{\Sigma}$ as

$$
\Sigma_{\mathrm{M}}=\boldsymbol{\Sigma} \otimes \mathbf{J}
$$

where $\mathbf{J}$ is a $n_{B S} \times n_{U T}$ matrix of ones. The entries of the $\Sigma$ matrix are defined by the variance profile function

$$
\varsigma(u, v)=(1+d(u, v))^{-\frac{\eta}{2}},
$$

where $u \in[0,1]$ and $v \in[0, K]$ are the normalized indexes for the BSs and the UTs respectively and $d(u, v)$ is the normalized distance between BS $u$ and user $v$.

According to [12], the asymptotic sum-rate capacity $C_{\mathrm{opt}}$ for the described model assuming a very large number of cells, is given by

$$
\begin{aligned}
& C_{\text {opt }}=\lim _{N \rightarrow \infty} \frac{1}{N} \mathcal{I}(\mathbf{x} ; \mathbf{y} \mid \mathbf{H}) \\
& =\lim _{N \rightarrow \infty} \mathbb{E}\left[\frac{1}{N} \sum_{i=1}^{N n_{B S}} \log \left(1+\frac{\tilde{\gamma}}{K n_{U T}} \lambda_{i}\left(\frac{1}{N} \mathbf{H H}^{\dagger}\right)\right)\right] \\
& =n_{B S} \int_{0}^{\infty} \log \left(1+\frac{\tilde{\gamma}}{K n_{U T}} x\right) d \mathrm{~F}_{\frac{1}{N} \mathbf{H H}^{\dagger}}(x) \\
& =n_{B S} \mathcal{V}_{\frac{1}{N} \mathbf{H H}^{\dagger}}\left(\frac{\tilde{\gamma}}{K n_{U T}}\right) \\
& =n_{B S} K n_{U T} \mathcal{V}_{\frac{1}{N} \mathbf{H}^{\dagger} \mathbf{H}}\left(\frac{\tilde{\gamma}}{K n_{U T}}\right)
\end{aligned}
$$

where $\tilde{\gamma}=K N P / \sigma^{2}=K N \hat{\gamma}$ is the system transmit power normalized by the receiver noise power $\sigma^{2}$. The term $\lambda_{i}(\mathbf{X})$ denotes the eigenvalues of matrix $\mathbf{X}$ and

$$
\begin{aligned}
\mathcal{V}_{\mathbf{X}}(\gamma) & \triangleq \mathbb{E}\left[\log \left(1+\gamma \lambda_{i}(\mathbf{X})\right)\right] \\
& =\int_{0}^{\infty} \log \left(1+\gamma \lambda_{i}(\mathbf{X})\right) d \mathrm{~F}_{\mathbf{X}}(x)
\end{aligned}
$$

is the Shannon transform with parameter $\gamma$ of a random square Hermitian matrix $\mathbf{X}$, where $\mathrm{F}_{\mathbf{X}}(x)$ is the cumulative function of the asymptotic eigenvalue distribution (a.e.d.) of matrix $\mathbf{X}$ [12]. For a rectangular Gaussian matrix $\mathbf{G} \sim \mathcal{C N}(\mathbf{0}, \mathbf{I})$ with $\beta$ being the columns/rows ratio, the a.e.d. of $\frac{1}{N} \mathbf{G}^{\dagger} \mathbf{G}$ converges almost surely (a.s.) to the nonrandom a.e.d. of the MarčenkoPastur law

$$
\mathcal{V}_{\frac{1}{N} \mathbf{G}^{\dagger} \mathbf{G}}(\gamma) \stackrel{\text { a.s. }}{\longrightarrow} \mathcal{V}_{\mathrm{MP}}(\gamma, \beta)
$$

$$
\begin{gathered}
\text { where } \mathcal{V}_{\mathrm{MP}}(\gamma, \beta)=\log \left(1+\gamma-\frac{1}{4} \phi(\gamma, \beta)\right) \\
+\frac{1}{\beta} \log \left(1+\gamma \beta-\frac{1}{4} \phi(\gamma, \beta)\right)-\frac{1}{4 \beta \gamma} \phi(\gamma, \beta)
\end{gathered}
$$

$$
\begin{aligned}
& \text { and } \phi(\gamma, \beta)= \\
& \left(\sqrt{\gamma(1+\sqrt{\beta})^{2}+1}-\sqrt{\gamma(1-\sqrt{\beta})^{2}+1}\right)^{2} .
\end{aligned}
$$

However, considering the described MIMO cellular channel the channel matrix contains elements of non-uniform variance. In this case, the a.e.d. of $\frac{1}{N} \mathbf{H} \mathbf{H}^{\dagger}$ is derived based on the analysis in [8] and using tools from the discipline of Free Probability. In this direction, $\frac{1}{N} \mathbf{H}^{\dagger} \mathbf{H}$ can be written as the sum of $K N n_{U T} \times K N n_{U T}$ unit rank matrices, i.e.

$$
\frac{1}{N} \mathbf{H}^{\dagger} \mathbf{H}=\sum_{i=1}^{N n_{B S}} \mathbf{h}_{i}^{\dagger} \mathbf{h}_{i}
$$

where $\mathbf{h}_{i} \sim \mathcal{C N}\left(\mathbf{0}, \mathbf{V}_{i}\right)$ denotes the $i$ th $1 \times K N n_{U T}$ row vector of $\frac{1}{\sqrt{N}} \mathbf{H}$, since the term $\frac{1}{N}$ has been incorporated in 
the unit rank matrices. The covariance matrix equals $\mathbf{V}_{i}=$ $\frac{1}{N}\left(\operatorname{diag}\left(\boldsymbol{\sigma}_{i}\right)\right)^{2}$, where $\operatorname{diag}\left(\boldsymbol{\sigma}_{i}\right)$ stands for a diagonal matrix with the elements of vector $\boldsymbol{\sigma}_{i}$ across the diagonal with $\boldsymbol{\sigma}_{i}$ being the $i$ th row of $\boldsymbol{\Sigma}_{\mathbf{M}}$. The unit-rank matrices $\mathbf{W}_{i}=\mathbf{h}_{i}^{\dagger} \mathbf{h}_{i}$ constitute complex singular Wishart matrices with one degree of freedom and their density according to [13] is

$$
\begin{aligned}
& f_{\mathbf{V}_{i}}\left(\mathbf{W}_{i}\right)=B_{\mathbf{V}_{i}}^{-1} \operatorname{det}\left(\mathbf{W}_{i}\right)^{1-K n_{U T} N} e^{-\operatorname{tr}\left(\mathbf{V}_{i}^{-1} \mathbf{W}_{i}\right)} \\
& B_{\mathbf{V}_{i}}=\pi^{K n_{U T} N-1} \operatorname{det}\left(\mathbf{V}_{i}\right) .
\end{aligned}
$$

It can be easily seen that if $\mathbf{V}_{i} \propto \mathbf{I}$, the matrices would be unitarily invariant and therefore asymptotically free [12, Example 2.46]. Although in our case $\mathbf{V}_{i}=\frac{1}{N}\left(\operatorname{diag}\left(\boldsymbol{\sigma}_{i}\right)\right)^{2}$, we assume that the asymptotic freeness still holds. Similar approximations have already been investigated in an information-theoretic context providing useful analytical insights and accurate numerical results [14],[15]. In this context, the R-transform of each unit rank matrix [12, Example 2.28] is given by

$$
\mathcal{R}_{\mathbf{h}_{\mathbf{i}}^{\dagger} \mathbf{h}_{\mathbf{i}}}(w)=\frac{1}{K N n_{U T}} \frac{\left\|\mathbf{h}_{i}\right\|^{2}}{1-w\left\|\mathbf{h}_{i}\right\|^{2}} .
$$

and the asymptotic R-transform of $\frac{1}{N} \mathbf{H}^{\dagger} \mathbf{H}$ is equal to the sum of the R-transforms of all the unit rank matrices [12, Th. 2.64]

$$
\begin{aligned}
\lim _{N \rightarrow \infty} \mathcal{R}_{\frac{1}{N} \mathbf{H}^{\dagger} \mathbf{H}}(w) & \simeq \lim _{N \rightarrow \infty} \sum_{i=1}^{N n_{B S}} \mathcal{R}_{\mathbf{h}_{\mathbf{i}}^{\dagger} \mathbf{h}_{\mathbf{i}}}(w) \\
& =\lim _{N \rightarrow \infty} \frac{1}{K N n_{U T}} \sum_{i=1}^{N n_{B S}} \frac{\left\|\mathbf{h}_{i}\right\|^{2}}{1-w\left\|\mathbf{h}_{i}\right\|^{2}} .
\end{aligned}
$$

Since the variance profile function of Equation (5) defines rectangular block-circulant matrix with $1 \times K$ blocks which is symmetric about $u=K v$, the channel matrix $\mathbf{H}$ is asymptotically row-regular [12, Definition 2.10] and thus the asymptotic norm of $\mathbf{h}_{i}$ converges to a deterministic constant for every $\mathrm{BS}$, i.e $\forall i$

$$
\lim _{N \rightarrow \infty}\left\|\mathbf{h}_{i}\right\|^{2}=\lim _{N \rightarrow \infty} \frac{1}{N} \sum_{j=1}^{K N n_{U T}} \varsigma_{i j}^{2}=\int_{0}^{K n_{U T}} \varsigma^{2}(u, v) d v
$$

where $\varsigma_{i j}$ is the $(i, j)$ th element of the $\Sigma_{\mathbf{M}}$ matrix. In addition, based on the row-regularity it can be seen that

$$
n_{B S} \int_{0}^{K n_{U T}} \varsigma^{2}(u, v) d v=\int_{0}^{n_{B S}} \int_{0}^{K n_{U T}} \varsigma^{2}(u, v) d u d v
$$

Therefore, the R-transform can be simplified to [12, Th. 2.31, Ex. 2.26]

$$
\begin{aligned}
& \lim _{N \rightarrow \infty} \mathcal{R}_{\frac{1}{N} \mathbf{H}^{\dagger} \mathbf{H}}(w) \\
& \simeq \frac{1}{K n_{U T}} \int_{0}^{n_{B S}} \frac{\int_{0}^{K n_{U T}} \varsigma^{2}(u, v) d v}{1-w \int_{0}^{K n_{U T}} \varsigma^{2}(u, v) d v} d u \\
& =\frac{1}{K n_{U T}} \frac{\int_{0}^{n_{B S}} \int_{0}^{K n_{U T}} \varsigma^{2}(u, v) d u d v}{n_{B S}-w \int_{0}^{n_{B S}} \int_{0}^{K n_{U T}} \varsigma^{2}(u, v) d u d v} \\
& =q\left(\boldsymbol{\Sigma}_{\mathbf{M}}\right) \frac{1}{1-\frac{K n_{U T}}{n_{B S}} w q\left(\boldsymbol{\Sigma}_{\mathbf{M}}\right)}=\mathcal{R}_{q\left(\mathbf{\Sigma}_{\mathbf{M}}\right) \frac{1}{N} \mathbf{G}_{\mathbf{M}}{ }^{\dagger} \mathbf{G}_{\mathbf{M}}}(w)
\end{aligned}
$$

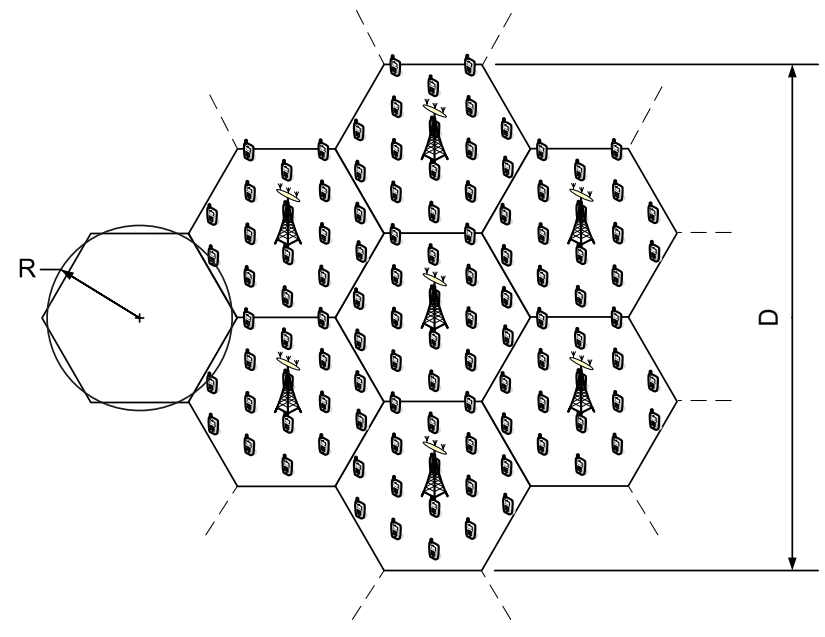

Fig. 1. Ground plan and dimensions of the cellular system comprising of BSs with multiple antennas and UTs distributed on a uniform planar grid. Parameters: $N=7, K=16$.

where $q\left(\boldsymbol{\Sigma}_{\mathbf{M}}\right) \triangleq\left\|\boldsymbol{\Sigma}_{\mathbf{M}}\right\|^{2} /\left(K N^{2} n_{U T} n_{B S}\right)$ is the Frobenius norm of the $\boldsymbol{\Sigma}_{\mathbf{M}}$ matrix $\left\|\boldsymbol{\Sigma}_{\mathbf{M}}\right\|^{2} \triangleq \operatorname{tr}\left\{\boldsymbol{\Sigma}_{\mathbf{M}}^{\dagger} \boldsymbol{\Sigma}_{\mathbf{M}}\right\}$ normalized with the matrix dimensions and

$$
\left\|\boldsymbol{\Sigma}_{M}\right\|^{2}=\operatorname{tr}\left\{(\boldsymbol{\Sigma} \otimes \mathbf{J})^{\dagger}(\boldsymbol{\Sigma} \otimes \mathbf{J})\right\}=\|\boldsymbol{\Sigma}\|^{2} n_{U T} n_{B S}
$$

Thus, it can be seen that $q\left(\boldsymbol{\Sigma}_{M}\right)=q(\boldsymbol{\Sigma})=\|\boldsymbol{\Sigma}\|^{2} /\left(K N^{2}\right)$. In the asymptotic case, $q(\boldsymbol{\Sigma})$ is given by $\lim _{N \rightarrow \infty} q(\boldsymbol{\Sigma})=$ $\frac{1}{K} \int_{0}^{K} \varsigma^{2}(u, v) d v$. Therefore, the a.e.d. of $\frac{1}{N} \mathbf{H}^{\dagger} \mathbf{H}$ follows a scaled version of the Marčenko-Pastur law and hence the Shannon transform of the a.e.d. of $\frac{1}{N} \mathbf{H}^{\dagger} \mathbf{H}$ can be approximated by

$$
\mathcal{V}_{\frac{1}{N} \mathbf{H}^{\dagger} \mathbf{H}}\left(\frac{\tilde{\gamma}}{K n_{U T}}\right) \simeq \mathcal{V}_{\mathrm{MP}}\left(q(\boldsymbol{\Sigma}) \frac{\tilde{\gamma}}{K n_{U T}}, K n_{U T}\right)
$$

As a result, the per-cell capacity is given by

$$
C_{\mathrm{opt}} \simeq n_{B S} K n_{U T} \mathcal{V}_{\mathrm{MP}}\left(q(\boldsymbol{\Sigma}) \frac{\tilde{\gamma}}{K n_{U T}}, K n_{U T}\right) .
$$

Equation (20) reveals a linear scaling of the MIMO sumrate capacity w.r.t. single-antenna sum-rate capacity, which comes into agreement with the already-existing results in the literature [2]. More specifically, when the number of UTs is large, the MIMO sum-rate capacity grows linearly with the number of BS antennas. What is more, as it can be seen in the next section, this linear growth continues to apply even when there is a single UT antenna.

\section{NUMERICAL RESUlTS}

This section presents and compares the analytical and simulations capacity results which have been produced by applying the Free Probability approach and running Monte Carlo simulations respectively. For the analytical results, the per-cell sum-rate capacity has been calculated using Equation (20). The coefficient $q(\boldsymbol{\Sigma})$ is calculated assuming that the UTs are positioned on a uniform planar grid (Figure 1). The percell sum-rate capacity has been plotted w.r.t. a variable cellular 


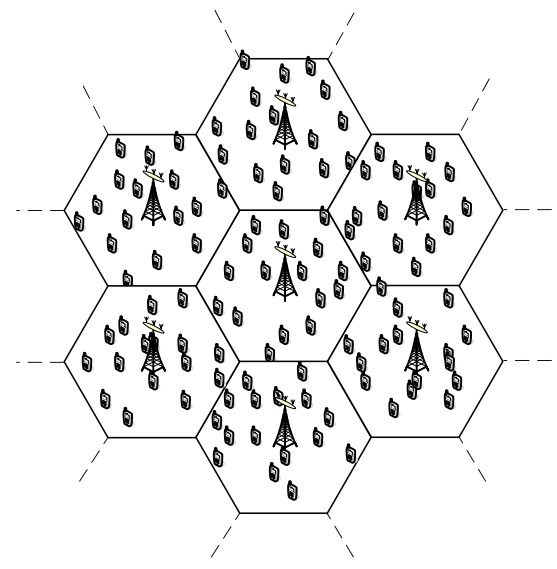

Fig. 2. Random snapshot of UT positions used for the Monte Carlo simulations. Parameters: $N=7, K=16$.

system coverage. The cellular coverage is varied by varying the edge length $D$ of the cellular coverage area (Figure 1). The analytical results have been verified by running Monte Carlo simulations over 1000 random instances of the system and by averaging the produced capacity results. More specifically, for each system instance the Gaussian complex matrix $\mathbf{G}_{\mathbf{M}}$ is constructed by randomly generating Gaussian i.i.d. c.c.s. fading coefficients. Similarly, the variance profile matrix $\boldsymbol{\Sigma}_{\mathbf{M}}$ is constructed by randomly placing the UTs according to the considered distribution in the coverage area of each cell (Figure 2) and by calculating the variance profile coefficients using Equations (5) and (4). After constructing the channel matrix, the sum-rate capacity is calculated by evaluating the formula in [1]

$$
C_{\text {opt }}=\frac{1}{N} \mathbb{E}\left[\log \operatorname{det}\left(\mathbf{I}_{N n_{B S}}+\frac{\hat{\gamma}}{n_{U T}} \mathbf{H H}^{\dagger}\right)\right]
$$

The simulation points are marked in Figures 3 and 4 using circle points.

\section{A. Multiple BS Antennas}

Figure 3 depicts the numerical results obtained by incorporating multiple antennas at the $\mathrm{BS}$ side. As it can be seen, the analytical results match the simulations and the linear scaling of sum-rate capacity w.r.t. the number of BS antennas $n_{B S}$ is established.

\section{B. Multiple UT Antennas}

In contrast with multiple BS antennas, incorporating multiple antennas at the UT side does not achieve a capacity gain. This can be intuitively explained by the fact that the sum-rate capacity grows linearly with $\min \left(n_{r}^{\text {tot }}, n_{t}^{\text {tot }}\right)$, where $n_{r}^{t o t}=N n_{B S}$ and $n_{t}^{t o t}=N K n_{U T}$ is the total number of receive and transmit antennas respectively in a MIMO GCMAC. Assuming $K \gg n_{B S} / n_{U T}$, it can be seen that $n_{t}^{\text {tot }} \gg n_{r}^{\text {tot }}$ and thus the sum-rate capacity grows linearly with the number of BS receive antennas $n_{B S}$.

This fact can also be explained using the derived closedforms. According to [12], the asymptotic of the Shannon

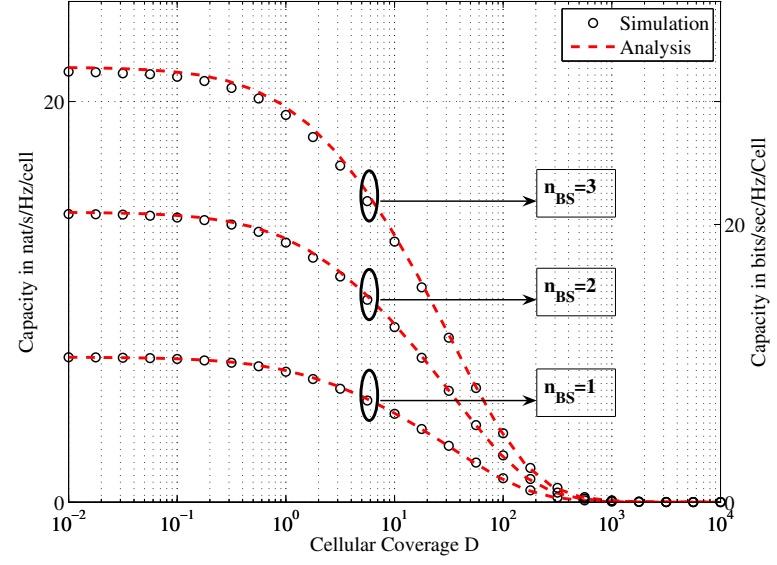

Fig. 3. Per-cell sum-rate capacity vs. the cellular coverage $D$ for uniformly distributed users. Parameter values: $\eta=2, K=16, N=19, \hat{\gamma}=$ $10, n_{B S}=[1,2,3]$

transform for $\beta>1$ is given by

$$
\lim _{\gamma \rightarrow \infty} \beta \mathcal{V}_{\mathrm{MP}}(\gamma, \beta)=\log (\beta \gamma)-(\beta-1) \log \left(\beta-\frac{1}{\beta}\right)-1 \text {. }
$$

Furthermore, the asymptotic sum-rate capacity for a very large number of UTs per cell converges to

$$
\lim _{\gamma, \beta \rightarrow \infty} \beta \mathcal{V}_{\mathrm{MP}}(\gamma, \beta)=\log (\beta \gamma)
$$

since $\lim _{\beta \rightarrow \infty}\left(1+\frac{1}{\beta}\right)^{\beta}=e$. In this direction, the per-cell capacity of Equation (20) can be simplified to

$$
\lim _{K \rightarrow \infty} C_{\text {opt }}=n_{B S} \log (q(\boldsymbol{\Sigma}) \tilde{\gamma})
$$

which is independent of the number of UT antennas $n_{U T}$. Hence, it can be seen that for $K$ UTs with a power constraint $P$ equally distributed over $n_{U T}$ antennas produce an equal per-cell capacity as $K$ single-antenna UTs with an individual power constraint of $P$.

\section{Practical Results}

The employed power-law path loss model of Equation (5) provides a variance profile coefficient as a function of the normalized distance $d(v)$. Similar path-loss models have been already utilized in the information-theoretic literature [8], [11], [3]. In order to apply the aforementioned results to realworld cellular systems, a reference distance $d_{0}$ is required to interconnect the normalized distance $d(v)$ and the actual distance $\hat{d}(v)$. Assuming that the power loss at the reference distance $d_{0}$ is $L_{0}$, the scaled variance profile function is given by

$$
\varsigma(d(v))=\sqrt{L_{0}\left(1+\hat{d}(v) / d_{0}\right)^{-\eta}} .
$$

In the context of a real-world macrocellular scenario, the typical parameters of Table I will be considered. Figure 4 depicts the per-cell capacity of the planar MIMO cellular system vs. the equivalent cell radius $R$ of the hexagonal cell, which is defined as the cell radius of a circular cell with equivalent coverage surface (Figure 1). 
TABLE I

VALUE/RANGE OF PARAMETERS USED FOR A TYPICAL MACROCELLULAR SCENARIO

\begin{tabular}{c|c|l} 
Parameter & & Value/Range \\
\hline Cell Radius & $R$ & $0.1-3 \mathrm{~km}$ \\
Reference Distance & $d_{0}$ & $1 \mathrm{~m}$ \\
Path Loss at ref. distance & $L_{0}$ & $38 \mathrm{~dB}$ \\
Path Loss Exponent & $\eta$ & 2 or 3.5 \\
UTs per cell & $K$ & 16 \\
UT Transmit Power & $P$ & $200 \mathrm{~mW}$ \\
Thermal Noise Density & $N_{0}$ & $-169 \mathrm{dBm} / \mathrm{Hz}$ \\
Channel Bandwidth & $B$ & $5 \mathrm{MHz}$ \\
\hline
\end{tabular}

\section{Discussion \& CONCLUSION}

This section analyses the presented results and tries to derive some insights on the practical performance of hyper-receiver cellular networks. In the model employed in this paper, the assumption of collocated users is alleviated by considering the more realistic scenario where the users are uniformly distributed across the cell's coverage area. The analysis of this model has shown that spectral efficiencies of up to 20 bits/s/Hz can be achieved for dense cellular networks assuming multicell joint processing and single BS antenna. Furthermore, by incorporating multiple antennas at the BS, a linear growth of the sum-rate capacity can be achieved w.r.t. the number of antennas. More importantly, it has been shown that increasing the number of UT antennas does not achieve higher capacities, which is convenient due to the size and power limitations of the mobile handsets.

However, it should be noted that the aforementioned MIMO results apply only to uncorrelated i.i.d. MIMO MAC channels, which is not always the case. For example, when the BS lies on high ground in a poor scattering environment, then correlation among the multiple receive antennas of a single BS can be considerable. These correlation effects can compromise the linear capacity scaling of the MIMO MAC channel. Another important assumption of the MIMO analysis is that there is no CSI available at the transmitters. This fact limits the sum-rate capacity, since the UT is unable to fully exploit the space diversity by optimizing the input signals over its transmit antennas. Such a transmitter optimization strategy could increase the throughput of the MIMO MAC channel. These scenarios will be considered in future work.

\section{ACKNOWLEDGMENT}

The work reported in this paper has formed part of the "Fundamental Limits to Wireless Network Capacity" Elective Research Programme of the Virtual Centre of Excellence in Mobile \& Personal Communications, Mobile VCE, www.mobilevce.com. This research has been funded by the following Industrial Companies who are Members of Mobile VCE - BBC, BT, Huawei, Nokia, Nokia Siemens Networks, Nortel, Vodafone. Fully detailed technical reports on this research are available to staff from these Industrial Members of Mobile VCE. The authors would like to thank Prof. G. Caire and Prof. D. Tse for the useful discussions.

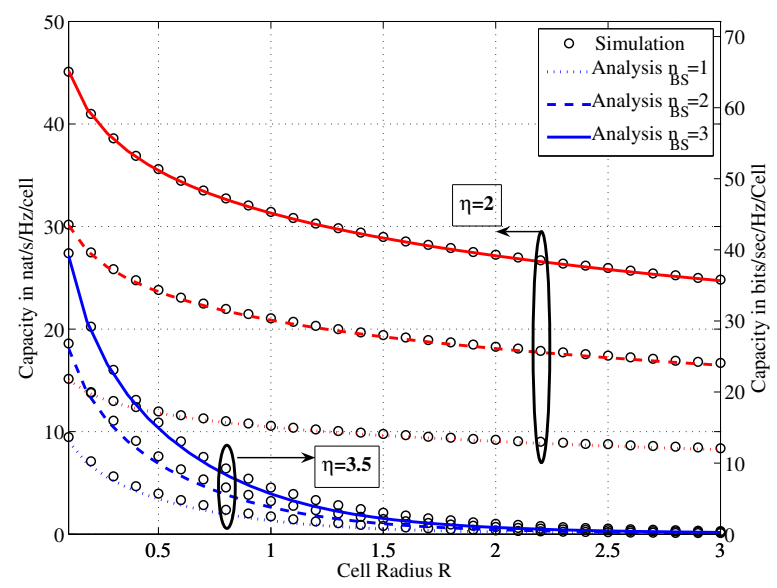

Fig. 4. Per-cell sum-rate capacity vs. the cell radius $R$ for a real-world MIMO cellular system. Parameters: $K=16, n_{B S}=[1,2,3], \eta=[2,3.5]$ and $P=0.2 \mathrm{~W}$.

\section{REFERENCES}

[1] I. E. Telatar, "Capacity of multi-antenna Gaussian channels," European Transactions on Telecommunications, vol. 10, no. 6, pp. 585-595, November 1999.

[2] W. Rhee and J. Cioffi, "On the capacity of multiuser wireless channels with multiple antennas," Information Theory, IEEE Transactions on, vol. 49, no. 10, pp. 2580-2595, October 2003.

[3] D. Aktas, M. Bacha, J. Evans, and S. Hanly, "Scaling results on the sum capacity of cellular networks with MIMO links," Information Theory, IEEE Transactions on, vol. 52, no. 7, pp. 3264-3274, July 2006.

[4] A. Wyner, "Shannon-theoretic approach to a Gaussian cellular multipleaccess channel," Information Theory, IEEE Transactions on, vol. 40, no. 6, pp. 1713-1727, Nov. 1994.

[5] O. Somekh and S. Shamai, "Shannon-theoretic approach to a Gaussian cellular multiple-access channel with fading," Information Theory, IEEE Transactions on, vol. 46, no. 4, pp. 1401-1425, July 2000.

[6] O. Somekh, B. Zaidel, and S. Shamai, "Sum rate characterization of joint multiple cell-site processing," Information Theory, IEEE Transactions on, vol. 53, no. 12, pp. 4473-4497, Dec. 2007.

[7] S. Shamai, O. Somekh, and M. Zaidel, "Multi-cell communications: An information theoretic perspective," in Joint Workshop on Communications and Coding, Donnini (Florence), Italy, 14-17 Oct 2004.

[8] N. A. Letzepis, "Gaussian cellular muptiple access channels," Ph.D. dissertation, Institute for Telecommunications Research, University of South Australia, December 2005.

[9] W. Yu, W. Rhee, S. Boyd, and J. Cioffi, "Iterative water-filling for gaussian vector multiple-access channels," Information Theory, IEEE Transactions on, vol. 50, no. 1, pp. 145-152, Jan. 2004.

[10] E. Visotsky and U. Madhow, "Space-time transmit precoding with imperfect feedback," Information Theory, IEEE Transactions on, vol. 47, no. 6 , pp. 2632-2639, Sep 2001.

[11] L. Ong and M. Motani, "The capacity of the single source multiple relay single destination mesh network," in IEEE International Symposium on Information Theory, Jul 2006, pp. 1673-1677.

[12] A. Tulino and S. Verdu, "Random matrix theory and wireless communications," Commun. Inf. Theory, vol. 1, no. 1, pp. 1-182, 2004.

[13] T. Ratnarajah and R. Vaillancourt, "Complex random matrices and applications," Computer \& Mathematics with Applications, vol. 50, no. 3-4, pp. 399-411, Aug 2005.

[14] M. Peacock, I. Collings, and M. Honig, "Asymptotic spectral efficiency of multiuser multisignature CDMA in frequency-selective channels," Information Theory, IEEE Transactions on, vol. 52, no. 3, pp. 11131129, Mar 2006

[15] W. Hachem, "Low complexity polynomial receivers for downlink CDMA," Signals, Systems and Computers, 2002. Conference Record of the Thirty-Sixth Asilomar Conference on, vol. 2, pp. 1919-1923 vol.2, Nov 2002. 